\title{
The effect of biofeedback training on anxiety in physical education Teachers short title: biofeedback training and PE teacher's anxiety
}

\begin{abstract}
Introduction: Stress and anxiety are inevitable parts of the teaching profession. The main purpose of this research was to examine the usefulness biofeedback training (BFB) as part of the Learning-Modification-Application (LMA) approach as a tool for physical education (PE) teachers to cope with their anxiety.

Material and methods: A total of 57 PE teachers, age 25-59 years $(M=40.5 \pm 9.02)$, volunteered to participate in the current study. The participants were graduate (MEd) students at an academic college in the southern part of Israel. The participants were randomly divided into three groups: LMA $(n=20)$, BFB relaxation games $(n=22)$; and Control $(n=15)$. Two questionnaires were used to measure anxiety: the StateTrait Anxiety Inventory (STAI) and the Sport Competition Anxiety Test (SCAT). The research protocol included 21 sessions, each lasting 60 minutes.

Results: The results of this research show that practicing the LMA approach has a positive effect on state anxiety, $\mathrm{t}(19)=2.92, \mathrm{p}=.009$ and trait anxiety $\mathrm{t}(19)=2.84$, $\mathrm{p}=.0101$.

Conclusion: It was found that PE teachers who practice the LMA approach experienced a stronger effect on state anxiety and sport competition anxiety compared to $\mathrm{PE}$ teachers practicing BFB relaxation games only. Practical implications for improving the coping skills of PE teachers with anxiety are discussed.
\end{abstract}

Keywords: biofeedback training, anxiety, physical education teacher
Volume 2 Issue 3 - 2017

\author{
Boris Blumenstein, ${ }^{1,2}$ Iris Orbach,' Ronen \\ Aaron, ${ }^{2}$ Yitzhak Weinstein, ${ }^{2}$ Daniel Moran ${ }^{2,3}$ \\ 'Ribstein Research Center,Wingate Institute, Israel \\ ${ }^{2}$ Givat Washington Academic College, Israel \\ ${ }^{3}$ Ariel University, Israel
}

Correspondence: Boris Blumenstein, Wingate Institute, Netanya 42902, Israel, Tel +97298639403, Fax +972986394I2, Email borisb@wingate.org.il

Received: June 19, 2017| Published: August 08, 2017
Abbreviations: LMA, learning modification application; PE, physical education; SCAT, sport competition anxiety test; W5SA, Wingate 5-step approach; STAI, state-trait anxiety inventory; SCAT, sport competition anxiety test; GSR, galvanic skin response; EDA, electro dermal activity; VCR, video camera recorded

\section{Introduction}

Stress is an unavoidable part of life and everyone has to deal with it. Teachers are exposed to heavy workloads and at least one-third of the teachers suffer from extreme stress and/or burnout. ${ }^{1} \mathrm{Kyriacou}^{2}$ defined teacher stress as: "the experience by teachers of unpleasant, negative emotions, such as anger, anxiety, tension, frustration and depression, resulting from some aspect of their work as a teacher". Causes of stress in teachers have been identified in a number of studies. ${ }^{3-5}$ The causes can be related to the person, to the teaching tasks, and to the work environment. As a result to these demands/ conditions, teachers may experience anxiety.

Although in some cases stress and anxiety may have a positive effect on the teacher, it is necessary to adopt coping strategies for managing these conditions. ${ }^{1,5}$ Grant $^{6}$ recommended developing workshops for teachers in which they will learn strategies such as relaxation, imagery, biofeedback training and cognitive skills, in addition to being taught about physical fitness and a healthy lifestyle. In these cases teachers will learn personal coping strategies to have at their disposal, in order to manage stress when it inevitably occurs. One of these effective coping strategies is biofeedback (BFB) training. ${ }^{7,8}$
BFB technology offers great promise for sport psychology, and specifically for psychological skills training for reducing competitive stress and performance enhancement. Research on the efficacy of BFB training has shown a positive effect in a variety of sport disciplines $^{8-10}$ and in other professionals such as music, ${ }^{11}$ the military ${ }^{12}$ and education. ${ }^{13}$ There is some evidence regarding the use of BFB training as a coping mechanism among teachers..$^{13}$ However, since most of the BFB training sessions occur in the laboratory, studies point to the fact that "one of the criticisms of biofeedback training has been the inability to transfer the learned response to performance in the real world". ${ }^{14}$

To overcome this limitation, two training models-the "Wingate 5-Step Approach" (W5SA) ${ }^{15}$ and the Learning-ModificationApplication (LMA) Approach ${ }^{7,9}$ were developed. The W5SA enables athletes to transfer the psycho-regulative skills acquired in the laboratory to real training and competition, utilizing testing and different simulative material. Thus, the goal of this method is to improve athletic self-regulation, optimize competitive behavior and improve athletic performance. Studies indicated the effectiveness of this method in populations such as athletes, ${ }^{7}$ physical education pupils ${ }^{16}$ and musicians. ${ }^{11}$ Later, the LMA approach was developed, which is an improved method of the W5SA. ${ }^{7,9}$

The LMA approach is composed of three dimensions: Learning, Modification and Application. Throughout the training program psychological skills are taught and practiced so that they will become more specific to sport and can be applied quickly, reliably and accurately. This process is accompanied by BFB support, which makes it fast and reliable. The athlete monitors his/her response in 
each stage and therefore is better able to understand his/her progress in achieving concrete goals on the way to performance enhancement. The skills are applied initially in the laboratory and then subsequently in preperformance and precompetitive routines. ${ }^{9}$

In the initial stages of the LMA approach, the athlete practices psychological strategies/techniques separately, while the final main goal is to put together the strategies into a mental training package. Moreover, the program is performed under various stress situations, which allows for the transfer of psychological skills to real

Table I Stress distraction scale used in the LMA approach? competitive events. The LMA approach includes a "stress distraction scale", which was developed based on applied work with a variety of athletes and sport disciplines (Table 1). The main goal of the scale is teach the athlete how to apply these psychological skills in real life. This scale used for the better and faster transfer of learned responses from laboratory to the sports ground. The LMA approach has been successfully applied to athletes in a variety of skills levels. ${ }^{9}$ The main purpose of the current research was to examine the usefulness of the LMA approach versus relaxation games with BFB as a tool to help physical education teachers cope with their daily stress.

\begin{tabular}{|c|c|c|c|c|}
\hline Stage & Duration & Location & Stress distraction level & Description \\
\hline \multirow{2}{*}{ Learning } & \multirow{2}{*}{$\approx 2$ months } & \multirow{2}{*}{ Laboratory } & $1 *$ & Ordinary laboratory settings \\
\hline & & & 2 & Positive/negative comments \\
\hline \multirow[t]{2}{*}{ Modification } & \multirow[t]{2}{*}{$\approx 2$ months } & \multirow[t]{2}{*}{$\begin{array}{l}\text { Laboratory and } \\
\text { training setting }\end{array}$} & 3 & $\begin{array}{l}\text { Performance under precise } \\
\text { demands (e.g., time, quality, } \\
\text { speed) }\end{array}$ \\
\hline & & & 4 & $\begin{array}{l}\text { Reward/punishment for } \\
\text { performance }\end{array}$ \\
\hline \multirow{3}{*}{ Application } & \multirow{3}{*}{$\approx 2$ months } & \multirow{3}{*}{$\begin{array}{l}\text { Laboratory, training } \\
\text { and competition } \\
\text { settings }\end{array}$} & 5 & $\begin{array}{l}\text { Performance under "true" } \\
\text { competition noise (competition } \\
\text { audio clips) }\end{array}$ \\
\hline & & & 6 & $\begin{array}{l}\text { Performance under "true" } \\
\text { competition sights (competition video clips) }\end{array}$ \\
\hline & & & 7 & Various combinations of levels 1-6 \\
\hline
\end{tabular}

*The scale ranges as follows: I-2 representing light stress; 3-4 moderate stress; and 5-7 representing high stress distractions.

\section{Material and methods}

\section{Participants}

A total of 57 participants, 25-59years $(\mathrm{M}=40.5, \mathrm{SD} \pm 9.02)$, volunteered to participate in the current study. The participants were graduate $\left(\mathrm{M}^{\mathrm{Ed}}\right)$ students at an academic college in the southern part of Israel. During the duration of the research all participants worked as physical education teachers in elementary schools. In addition, all participants were formerly involved in competitive sport. The participants were randomly divided into three groups: LMA $(n=20)$, BFB Games $(\mathrm{n}=22)$ and Control $(\mathrm{n}=15)$.

\section{Procedure}

The three groups completed the State-Trait Anxiety Inventory $(\mathrm{STAI})^{17}$ and Sport Competition Anxiety Test (SCAT) ${ }^{18}$ questionnaires before and after the research procedures. The research protocol consisted of 21 sessions, each lasting for 60 minutes once a week. The sessions were divided into 3 parts, 7 sessions in each.

\section{Part I}

i. The LMA group practiced relaxation ${ }^{19}$ with GSR/EDA (Galvanic Skin Response/Electrodermal Activity) BFB, while the goal was to achieve a resistance reading difference (delta, $\Delta$ ) of $50 \mathrm{~K} \Omega$. After achieving the goal the participants were exposed to stress level 1-2 according to Table 1.

ii. The BFB Games group practiced relaxation skill similar to the LMA group's protocol while using multimedia BFB games (i.e., sensitivity levels 8-10).

iii. The Control group continued their academic lessons as usual.

\section{Part II}

i. The LMA group practiced relaxation skills with GSR/EDABFB while being exposed to stress level 3-4 according to Table 1 .

ii. The BFB Games group practiced relaxation skills similar to the LMA group's protocol while using multimedia BFB games (i.e., sensitivity levels 6-7).

iii. The Control group continued their academic lessons as usual.

\section{Part III}

i. The LMA group practiced relaxation skills with GSR/EDABFB while being exposed to stress levels 5-7 according to Table 1, which was modified to suit the teacher's profession (e.g., pictures of lecture, noises of classroom).

ii. The BFB Games group practiced similarly to the LMA group 
relaxation skill while using multimedia BFB games (i.e., history, levels 8-10).

iii. The Control group continued their academic lessons as usual.

\section{Instruments}

The computer software and BFB apparatus used to monitor and measure the physiological components was the Mind life biofeedback device (Pro Relax by Mind life Solutions Ltd.) which monitors skin conductance via two 5-mm-diameter electrodes connected with Velcro bands. ${ }^{20}$ The electrodes are connected to a sensor box and the raw data is transmitted via IR connection to the receiver box. The isolated skin conductance coupler applies a constant $0.5 \mathrm{~V}$ potential across the electrode pair. The sample rate is 10 samples per second. This BFB system can simultaneously or separately display all channels on graph or on bar graph screens.

In addition, three animated BFB multimedia relaxation games of the Pro Relax software using the themes nature, fish and history were used. In these dynamic games participants used relaxation and concentration skills to move an object (e.g., fish, a person) forward from the left to right side and to transform the object to other forms. The game's sensitivity can be changed from 1 to 10 (i.e., difficulteasy). The games are accompanied with special relaxation music. Other equipment devices include two sets of TV-VCR camera systems, video camera recorded (VCR), and audio devices. Finally, two questionnaires were used to assess anxiety: the STAI ${ }^{17}$ and SCAT. $^{18}$

\section{Results}

A mixed model ANOVA on baseline data indicated no significant differences among the three research groups at the outset of the study. Thus a one-way MANOVA for after treatment data was applied to the three dependent variables (TA, SA and SCAT). The results revealed a significant multivariate main effect for Groups: Wilks Lambda $=.787$, $F(6)=2.209, p=.048$. A series of one-way ANOVAs on each of the three dependent variables (TA_after, SA_after, SCAT_after) was conducted as a follow-up test to the MANOVA. The results indicated a significant main group effect for the SA_after $(\mathrm{F}(2,54)=4.25, \mathrm{p}=.019)$ and SCAT_after $(\mathrm{F}(2,54)=3.58, \mathrm{p}=.035)$. A Tukey post-hoc analysis revealed that the LMA was significantly lower $(\mathrm{p}=.014)$ on SA_after compared to Games $(\mathrm{M}=13.35, \mathrm{SD} \pm 8.56$ and $\mathrm{M}=22.23, \mathrm{SD} \pm 11.05$, respectively) (Table 2).

Similar results were found on SCAT_after. A Tukey post-hoc analysis revealed that the LMA was significantly lower $(\mathrm{p}=.035)$ on SCAT_after compare to Games $(\mathrm{M}=24.85, \mathrm{SD} \pm 6.31$ and $\mathrm{M}=28.77$, $\mathrm{SD} \pm 4.71$, respectively) (Table 2 ). In addition, a t-test was conducted for TA, SA, and SCAT to compare the treatment effect for each group. Significant differences were found only for the LMA group on TA: $\mathrm{t}(19)=2.84, \mathrm{p}=.0101$ and SA: $\mathrm{t}(19)=2.92, \mathrm{p}=.009$. The LMA group experienced a decrease in TA (M_before $=34.05, \mathrm{SD} \pm 7.03$, M_after $=31.30, \mathrm{SD} \pm 7.80$ ), and $\mathrm{SA}$ (M_before $=16.95, \mathrm{SD} \pm 10.93 \mathrm{M}$ after $=13.35, \mathrm{SD} \pm 8.56$ ) (Table 2).

Table 2 Comparison of before and after treatment measurements $(M \pm S D)$ for the three research groups on TA, trait anxiety; SA; state anxiety; SCAT, sport competitive anxiety test

\begin{tabular}{lllllll}
\hline Questionnaire group & TA & & SA & \multicolumn{2}{c}{ SCAT } \\
\hline LMA $(\mathrm{n}=20)$ & Before & After & Before & After & Before & After \\
Games $(\mathrm{n}=22)$ & $34.05 \pm 7.0^{* 3}$ & $31.30 \pm 7.8^{* 3}$ & $16.95 \pm 10.9^{* 4}$ & $13.35 \pm 8.6^{* 1,4}$ & $26.30 \pm 6.7$ & $24.85 \pm 6.3^{* 2}$ \\
Control $(\mathrm{n}=15)$ & $36.77 \pm 7.7$ & $36.10 \pm 8.8$ & $22.05 \pm 10.4$ & $22.23 \pm 11.0^{* 1}$ & $29.45 \pm 5.3$ & $28.77 \pm 4.7 * 2$ \\
\hline
\end{tabular}

\section{Discussion}

The main purpose of this research was to examine the usefulness of the LMA approach as a tool for helping physical education teachers cope with their daily stress. Previous studies mainly focused on using different biofeedback modalities to reduce athletic anxiety and enhance performance among elite athletes. ${ }^{20}$ The main finding of the current study was that using an intervention package which included BFB training (i.e., the LMA approach) enabled the physical education teachers to reduce their anxiety. For this purpose physical education teachers were exposed to the LMA approach for 21 weeks while measuring different kinds of anxiety (i.e., the LMA group). The second group played BFB multimedia relaxation games (i.e., Games) for the same number of weeks. The treatment was given to the LMA and Games groups in addition to their regular academic lessons. Finally, the third group continued their academic lessons as part of their daily routine (i.e., Control). The overall results of this study indicated that PE teachers in the LMA group exhibited the greatest improvement in reducing anxiety compared to the Games group. The results validated our basic hypothesis derived from previous research ${ }^{7}$ and from applications of the LMA with elite athletes. ${ }^{7,9}$
The results of the current study show that practice with the LMA approach leads to a decrease in trait and state anxiety levels. The main feelings of anxiety experienced are worry, nervousness, and apprehension, which can contribute to a feeling of loss of control and depression in the long run, as well as a decrease in physical health. ${ }^{21,22}$ Similar to the known interventions to reduce anxiety, such as physical activity, relaxation training, yoga and a variety of psychological interventions, the LMA approach can be used as an effective means to reduce and control physical education teachers' anxiety. ${ }^{7,23,24}$

In addition, it was found that physical education teachers who practice the LMA approach experienced a stronger effect on state anxiety and sport competitive anxiety compared to physical education teachers practicing only BFB relaxation games. Previous studies that investigated the effect of practicing with educational computer games on learning process exhibited positive effects. This effect was related to students' motivation to learn ${ }^{25,26}$ and to an environment which was perceived as safe for exploration and experimentation. ${ }^{27}$

\section{Conclusion}

The current study has shown that BFB relaxation games have an 
effect on state anxiety and competition anxiety. However, this effect was not as strong as the shown effect after using LMA approach. The LMA approach is a multifaceted psychological program that integrates BFB training with other psychological strategies into one intervention package. A main orientation of the LMA approach has been to reduce and regulate anxiety specifically in sport competition. ${ }^{7}$ For this reason, practice with the LMA approach was suggested under seven stress distractions/situations, which allows for the transfer of psychological skills to real-life situations. Furthermore, the LMA approach has a clear and rational structure that is based on the theory and methodology of sport training, ${ }^{28}$ which enables a logical and organized treatment for performance enhancement. Participants in this study had been engaged in competitive sport in the past. The results of our study are in line with research suggesting that physical education teachers have a better control of anxiety.,4 This is partly due to their knowledge about using cognitive strategies, acquired during their previous experience as competitive athletes. ${ }^{29}$ Limitation of this study might be related to the differences in professional experience of the participants due to age difference (25-59years old). In addition, all participants were physical education graduate students, a fact which may have an effect on the results. Future studies should focus on the effect of the LMA approach on anxiety among teachers in different academic disciplines.

\section{Acknowledgements}

None.

\section{Conflict of interest}

The author declares no conflict of interest.

\section{References}

1. Dick RV, Wagner U. Stress and strain in teaching: A structural equation approach. Br J Educ Psychol. 2001;71(Pt 2):243-259.

2. Kyriacou C. Teacher stress: Directions for future research. Educational Review. 2001;53(1):27-35.

3. Adams E. A proposed causal model of vocational teacher stress. Journal of Vocational Education and Training. 2001;53(2):223-246.

4. Brown M, Ralph S. Teacher stress and school improvement. Improving Schools. 2002;5(2):55-65.

5. Wilson V, Hall J. Running twice as fast? A review of the research literature on teachers' stress. Scottish Educational Review. 2002;34(2):175-187.

6. Grant GF. Stress factors among college educators. Master's Thesi, Canada: Brock University; 1991. 105 p.

7. Blumenstein B, Orbach I. Biofeedback for sport and performance enhancement. USA: Oxford Handbooks Onlin; 2014.

8. Edmonds WA, Tenenbaum G. Case studies in applied psychophysiology: Neurofeedback and biofeedback treatments for advances in human performance. USA: Wiley-Blackwell; 2012. 342 p.

9. Blumenstein B, Orbach I. Psychological skills in sport: Training and application. USA: Nova Science Publishers; 2012.

10. Perry FD, Shaw L, Zaichkowsky L. Biofeedback and neurofeedback in sports. Bio feedback. 2011;39(3):95-100.

11. Bazanova OM. Simultaneous alpha EEG enhancing and frontal's muscle EMG decreasing: Biofeedback training for musical peak performance. In:
Edmonds WA, et al. editors. Case studies in applied psychophysiology: Neurofeedback and biofeedback treatments for advances in human performance. UK: 2012. p. 71-90.

12. Oded Y. Biofeedback-based mental training in the military-the "mental gym" project. Biofeedback. 2011;39(3):112-118.

13. Walton JM. Biofeedback: A proposed model for the treatment of teacher anxiety. Journal of Counseling and Development. 2011;60(1):59-62.

14. Crews D, Lochbaum M, Karoly P. Self-regulation: Concepts, methods and strategies in sport and exercise. In: Singer R, et al. editors. Handbook of sport psychology 2nd ed. USA: J Wiley \& Sons; 2011. p. 566-581.

15. Blumenstein B, Eli BM, Tenenbaum G. A five-step approach to mental training incorporating biofeedback. Human Kinetics. 1997;11(4):440-453.

16. Eli BM, Blumenstein B. The effect of extra-curricular mental training with biofeedback on short running performance of adolescent physical education pupils. European Physical Education Review. 2004;10:123134

17. Spielberger CD, Gorsuch RL, Lushene RE. Manual for the State-Trait Anxiety Inventory. USA: Consulting Psychologists Press; 1970.

18. Martens R, Vealey RS, Burton D. Competitive anxiety in sport. UK: Human Kinetics; 1990. 277 p.

19. Jacobson E. Progressive relaxation. USA: University of Chicago Press; 1938.

20. Blumenstein B, Orbach I. The road to Olympic medal. In: Edmonds WA, et al. editors. Case studies in applied psychophysiology: Neurofeedback and biofeedback treatments for advances in human performance. UK: Wiley-Blackwell; 2012. p. 120-132.

21. Huberty TJ. Best practices in school-based interventions for anxiety and depression. In: Thomas A, et al. editors. Best practices in school psychology Vol. 5. USA: National Association of School Psychologists; 2008. p. 1473-1486.

22. Spielberger CD. Theory and research on anxiety. In: Spielberger CD, editors. Anxiety and behavior. USA: Academic Press; 1966. p. 3-22.

23. Kirkwood G, Rampes H, Tuffrey V, et al. Yoga for anxiety: A systematic review of the research evidence. Br J Sports Med. 2005;39(12):884-891.

24. Salmon P. Effects of physical exercise on anxiety, depression and sensitivity to stress: A unifying theory. Clin Psychol Rev. 2001;21(1):3361.

25. Ke F. Computer games application within alternative classroom goal structures: Cognitive, metacognitive and affective evaluation. Educational Technology Research and Development. 2008;56(5-6):539-556.

26. Rieber L. Multimedia learning in games, simulations, and microworlds In: Mayer RE, editors. The Cambridge Handbook of Multimedia Learning. USA: Cambridge University Press; 2005. p. 549-567.

27. Kinzie M, Joseph D. Gender differences in game activity preferences of middle school children: Implications for educational game design. Educational Technology Research and Development. 2008;56(5-6):643663.

28. Carrera M, Bompa T. Theory and methodology of training: General perspectives. In: Blumenstein B, editors. Psychology of sport training. UK: Meyer \& Meyer Sport; 2007. p. 19-39.

29. Salavera C, Antonanzas JL, Noe R, et al. Emotion and anxiety in teachers: Research of teaching physical education. Procedia-Social and Behavioral Sciences. 2014;132:577-581. 